\title{
Zukunft gestalten
}

\section{Lattrich}

\section{Der Nachwuchs der DGGG (Deutsche Gesellschaft für Gynäkologie und Geburtshilfe) traf sich vom 27.-29. März 2009 in Zell am See zum ersten „Gyn\&Ski“.}

Alle Mitglieder in der DGGG, die sich in der Weiterbildung befinden, sind Mitglieder des Jungen Forums. Es fängt die Probleme und Wünsche dieser Gruppe auf und artikuliert sie innerhalb der DGGG. Das funktioniert umso besser, je mehr Ärztinnen und Ärzte in der Weiterbildung ihre Gedanken diskutieren. Es zeigte sich, dass diese Möglichkeit in der Kombination mit Skifahren für fast 60 Kolleginnen und Kollegen besonders aktiv wahrgenommen wurde.

Es fand ein intensiver Austausch zur Zukunft der Weiterbildung statt. Die Vereinbarkeit von Weiterbildung und Familie, sowie die empfundene geringe Qualität der Weiterbildung dominierten die Gespräche.

Zudem war die Notwendigkeit Nachwuchs für unser Fach zu akquirieren offensichtlich. Trotz der gleichbleibenden Anzahl erfolgreicher Facharztprüfungen pro Jahr gibt es durch die Arbeitszeitgesetzgebung und die Veränderungen der organisatorischen Strukturen im Gesundheitssystem einen zunehmenden Bedarf an Ärztinnen und Ärzten.

Teilnehmer des Gyn\&Ski Skirennens in Zell am See (Bild: C. Lattrich).

Wie treffen Medizinstudenten die Entscheidung für die Weiterbildung zum Facharzt für Gynäkologie und Geburtshilfe?

$\checkmark$

Eine Möglichkeit ist es, dass die Berufswahl von vornherein feststeht. In diesem Fall ist keine positive Einflussnahme nötig, aber es sollte verhindert werden, dass negative Erfahrungen zu einem Umdenken führen. Doch häufig fällt die Entscheidung erst später. Sie wird beeinflusst durch die Qualität der Vorlesungen und Praktika, die für das Fach begeistern können. Den maßgeblichen Einfluss auf die Wahl der Fachrichtung haben jedoch die praktischen Erfahrungen: Famulaturen oder das Praktische Jahr. In diesem Zeitraum lernt die Studentin/der Student nicht nur das Fach mit all seinen Facetten kennen, sondern hat erstmals Kontakt zur momentanen Realität des Berufes. Das heißt, es wird ihr/ihm in dieser Zeit nicht nur theoretisches und praktisches Wissen des Faches vermittelt, sondern auch wie das Arbeitsumfeld der Ärzte strukturiert ist. Die Anforderungen und die möglichen Bewältigungsstrategien werden vorgelebt.

Sicher kann durch ein kollegiales Arbeitsumfeld, die Möglichkeit der individuellen

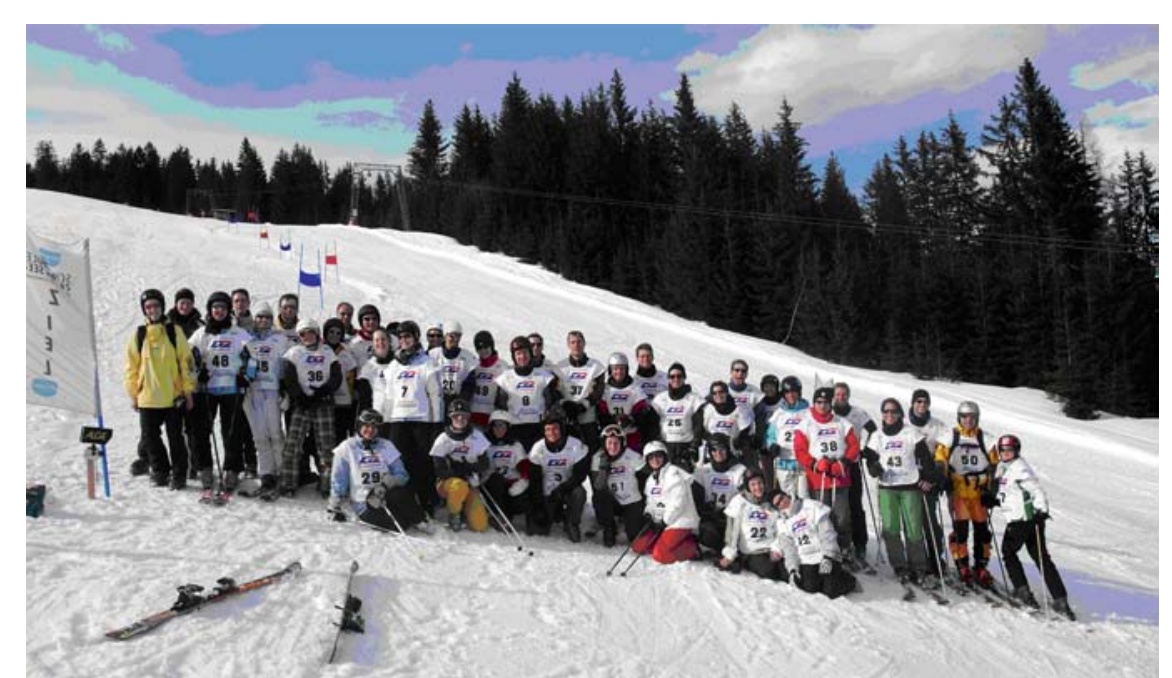

Die nächste Mitgliederversammlung des Jungen Forums findet im Rahmen des DAGG Weiterbildungkongresses vom 1.3. Oktober 2009 in Berlin statt. Interessierte sind herzlich willkommen!

Weitere Informationen zum Jungen Forum befinden sich auf der Homepage der DGGG (www.dggg.de).

\section{Dr. C. Lattrich}

Klinik für Frauenheilkunde und Geburtshilfe der Universität Regensburg am Caritas Krankenhaus St. Josef E-Mail: claus.lattrich@klinik.uni-r.de 